\title{
Clase social, bienestar y afectividad general durante la pandemia de COVID-19 en Iberoamérica
}

\section{Social class, well-being, and general affectivity during the COVID-19 pandemic in Ibero-America}

Ginés Navarro-Carrillo", Lander Méndez², Huseyin Cakal², Darío Páez ${ }^{2}$, José J. Pizarroº, ${ }^{10}$, Larraitz Zumeta², Marcela Gracia-Leiva², Silvia da Costa², Nekane Basabe², Saioa Telletxea², Gisela Delfino ${ }^{4}$, Elza Techio ${ }^{5}$, Anderson Mathias ${ }^{2}$, Carolina Alzugaray-Ponce ${ }^{6}$, Loreto Villagrán ${ }^{7}$, Gonzalo Martínez-Zelaya ${ }^{8}$, Marian Bilbao ${ }^{9}$, Anna Wlodarczyk ${ }^{10}$, Wilson López-López ${ }^{11}$, Laura Alfaro-Beracoechea ${ }^{12}$, Manuel. L. Ibarra ${ }^{13}$, Catarina Carvalho ${ }^{14}$, Isabel. R. Pinto ${ }^{14}$, Emilio Moyano-Díaz ${ }^{15}$, Agustín Espinosa ${ }^{16}$ y Rosa. M. Cueto ${ }^{16}$.

${ }^{1}$ Universidad de Jaén, España. ${ }^{2}$ Universidad del País Vasco, España. ${ }^{3}$ Universidad de Keele, Reino Unido. ${ }^{4}$ Pontificia Universidad Católica Argentina, Argentina. 5Universidad Federal de Bahía, Brasil, 6 Universidad Santo

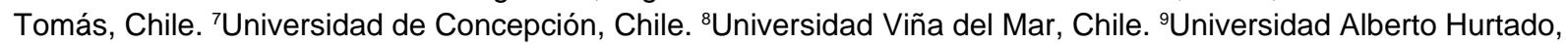
Chile. ${ }^{10}$ Universidad Católica del Norte, Chile. ${ }^{11}$ Pontificia Universidad Javeriana, Bogotá, Colombia. ${ }^{12}$ Universidad de Guadalajara, México. ${ }^{13}$ Universidad Autónoma del Estado de México, México. ${ }^{14}$ Universidad de Oporto, Portugal. ${ }^{15}$ Universidad de Talca, Chile. ${ }^{16}$ Pontificia Universidad Católica del Perú.

\section{Resumen}

La actual pandemia de la COVID-19 ha supuesto un marcado incremento de las brechas sociales preexistentes. En esta investigación analizamos las relaciones de diferentes facetas de clase social con el bienestar y afectividad general a lo largo de un total de 8 países de la región cultural iberoamericana. Asimismo, también pusimos a prueba el potencial rol mediador del riesgo percibido relativo a la COVID-19 y de la eficacia colectiva percibida. Los resultados obtenidos (a) revelaron que una clase social más baja se relacionó con un menor bienestar y afecto positivo, así como con un mayor afecto negativo; (b) confirmaron que la clase social subjetiva constituye un mejor predictor del bienestar y afectividad general que el nivel educativo (i.e., indicador de clase social objetiva); y (c) mostraron que los efectos de la clase social subjetiva en el bienestar/afectividad general se daban de forma indirecta a través del riesgo percibido y la eficacia colectiva. Estos hallazgos confirman la existencia de diferencias en bienestar y afectividad general en función de la clase social durante la pandemia, al tiempo que permiten avanzar en la comprensión de los mecanismos psicosociales subyacentes.
Abstract
The current COVID-19 pandemic has sharply increased preexisting social divides. In this research, we analyzed the relationships of different facets of social class with well-being and affectivity across 8 lbero-American countries. Moreover, we also tested the potential mediating role of perceived risk of COVID-19 and perceived collective efficacy. The results obtained (a) revealed that a lower social class was related to reduced well-being and positive affect as well as to greater negative affect, (b) confirmed that subjective social class was a better predictor of well-being and affectivity than educational level (i.e., an objective indicator of social class), and (c) showed that subjective social class was indirectly linked to well-being/affectivity via its effects on the perceived risk of COVID-19 and perceived collective efficacy. These findings support the existence of differences in well-being and affectivity as a function of social class during the pandemic, while advancing our understanding of underlying psychosocial mechanisms.

Keywords: COVID-19; subjective social class; well-being, affectivity; perceived risk; collective efficacy.

Palabras clave: COVID-19; clase social subjetiva; bienestar, afectividad; riesgo percibido; eficacia colectiva.

Cómo citar: Navarro-Carrillo, G., Méndez, L., Cakal, H., Páez, D., Pizarro, J. J., Zumeta, L., Gracia-Leiva, M., da Costa, S., Basabe, N., Telletxea, S., Delfino, G., Techio, E., Mathias, A., Alzugaray-Ponce, C., Villagrán, L., Martínez-Zelaya, G., Bilbao, M., Wlodarczyk, A., López-López, W.,...Cueto, R. M. (2021). Clase social, bienestar y afectividad general durante la pandemia de la COVID-19 en Iberoamérica. Escritos de Psicología - Psychological Writings, 14(2), 84-95. https:// doi.org/10.24310/espsiescpsi.v14i2.13608

Correspondencia: Lander Méndez. Universidad del País Vasco/Euskal Herriko Unibertsitatea. Avenida Tolosa, 70. 20018 Donostia/San Sebastián E-mail: lander.mendez@ehu.eus 
GINÉS NAVARRO-CARRILLO, LANDER MÉNDEZ, HUSEYIN CAKAL, DARÍO PÁEZ, JOSÉ J. PIZARRO, LARRAITZ ZUMETA, MARCELA GRACIA-LEIVA, SILVIA DA COSTA, NEKANE BASABE, SAIOA TELLETXEA, GISELA DELFINO, ELZA TECHIO,

ANDERSON MATHIAS, CAROLINAALZUGARAY-PONCE, LORETO VILLAGRÁN, GONZALO MARTÍNEZ-ZELAYA, MARIAN BILBAO,

ANNA WLODARCZYK, WILSON LÓPEZ-LÓPEZ, LAURA ALFARO-BERACOECHEA, MANUEL. L. IBARRA, CATARINA CARVALHO, ISABEL. R. PINTO, EMILIO MOYANO-DÍAZ, AGUSTÍN ESPINOSA, ROSA. M. CUETO

\section{Introducción}

La actual crisis global desatada por la COVID-19, que tiene como base el virus SARS-CoV-2, adquirió la dimensión de pandemia el 11 de marzo de 2020 (Organización Mundial de la Salud, 2020). Desde entonces, sus efectos, agravados por la rápida propagación del virus, se han manifestado de forma particularmente acusada a lo largo de todos los continentes, constituyendo una grave amenaza para la salud pública y la actividad económica. A pesar de que existen voces que han subrayado el carácter generalizado del impacto de la COVID-19, trabajos recientes sostienen que la actual crisis pandémica, tal y como también sucedió en otros contextos de crisis (Boyce et al., 2018), no afectaría a toda la ciudadanía por igual. Así pues, se ha propuesto que la actual pandemia acrecentará las brechas sociales preexistentes, siendo las personas pertenecientes a clases sociales más desfavorecidas, en comparación con las de clases más altas, las que manifestarán una mayor vulnerabilidad a su impacto (Rodríguez-Bailón, 2020). En este marco general, singularizado por niveles crecientes de disparidad socioeconómica, las diferencias de clase se observan con más nitidez (Moya \& Fiske, 2017). Por consiguiente, resulta de gran relevancia considerar el papel de la clase social durante la pandemia de COVID-19.

Esta investigación persigue analizar la asociación entre la clase social y diferentes indicadores de bienestar y afectividad durante la pandemia de COVID-19. Asimismo, también se analiza una vía a través de la cual la clase social de pertenencia podría conectarse con el bienestar y la afectividad. Más específicamente, examinamos el potencial papel mediador desempeñado de forma simultánea por el riesgo percibido referido a la COVID-19 y la sensación de eficacia colectiva a la hora de enfrentar las consecuencias de dicha crisis.

\section{Clase social y bienestar/afectividad}

La clase social, también denominada estatus socioeconómico, es conceptualizada en el ámbito psicosocial atendiendo tanto a la capacidad de acceso a recursos materiales -evaluada por medio de indicadores como el nivel de ingresos o la formación educativa- como a la percepción subjetiva del individuo con respecto a su posición en la jerarquía social -aspecto evaluado a través de "escaleras sociales" pictóricas (p.ej., Kraus et al., 2009). Un amplio volumen de investigaciones empíricas ha confirmado que la clase social de las personas impregna buena parte de su comportamiento (véase Manstead, 2018). Una de las líneas clásicas de estudio tiene que ver con la asociación existente entre la clase social y el bienestar.

Numerosos trabajos empíricos han mostrado que la clase social se relaciona positivamente con el bienestar. Los estudios y meta-análisis muestran un patrón de asociaciones positivas de leves a moderadas de la clase social con la salud mental (Zell et al., 2018) y el bienestar subjetivo (Yu \& Blader, 2020). Sin embargo, cabe mencionar que dichas relaciones suelen ser menos intensas en el caso de la clase social objetiva (p.ej., ingresos, educación; Diener \& Oishi, 2000; Diener et al., 2003). Según varios estudios (p.ej., Boyce et al., 2010; Navarro-Carrillo et al., 2020), la clase social subjetiva parece explicar mejor que la clase social objetiva posibles diferencias en los niveles de bienestar, toda vez que muestra correlatos más altos con distintos indicadores de bienestar y salud y, además, dichas relaciones se dan incluso controlando diferentes facetas objetivas de clase social (p.ej., Cundiff \& Matthews, 2017). Un meta-análisis reciente (Tan et al., 2020) proporcionó respaldo adicional a dicha idea: la magnitud de la relación entre la clase social subjetiva y el bienestar $(r=.22)$ fue superior a la de la asociación entre la clase social objetiva y el bienestar $(r=.16)$.

En este trabajo nos focalizamos en el papel de la clase social subjetiva en relación con el bienestar y afectividad durante la pandemia de COVID-19. No obstante, también evaluaremos el nivel educativo, uno de los indicadores de clase social objetiva más representativos a la luz de su capacidad para conducir a recursos económicos y posiciones laborales valoradas, así como de predecir diferentes tendencias comportamentales (Snibbe \& Markus, 2005). De esta manera, se podrá determinar si la clase social subjetiva predice positivamente el bienestar y la afectividad más allá de características sociodemográficas básicas (i.e., género y edad) y del nivel educativo.

\section{El potencial rol mediador del riesgo percibido y la eficacia colectiva}

El aumento de las disparidades socioeconómicas durante la pandemia está poniendo de relieve la especial vulnerabilidad de las personas más desfavorecidas (Pons-Valls, 2020). A las ya habituales condiciones materiales inestables que presentan las personas de clases desfavorecidas (Kraus et al., 2012), se suma el aumento de su inestabilidad económica y el desempeño de actividades laborales de mayor exposición física (Afridi \& Block, 2020; Blow, 2020). Cabe argumentar, pues, que las personas de clases más bajas podrían mostrar una mayor percepción de riesgo respecto a los efectos de la COVID19. Investigaciones previas, en el contexto de otras crisis (p.ej., financieras), revelaron que pertenecer a 
una clase social más desfavorecida era indicativo de mayores niveles de riesgo (económico) percibido (Alonso-Ferres et al., 2020). Asimismo, se ha corroborado el efecto nocivo de la percepción de riesgo -tanto durante la COVID-19 (Han et al., 2021) como en otras situaciones de crisis (Navarro-Carrillo et al., 2019)- sobre diferentes indicadores de bienestar y salud.

La explicación de la asociación entre la clase social y el bienestar/afectividad podría descansar también en el mayor estatus y poder asociados a las clases altas (Yu \& Blader, 2020). En este sentido, la vulnerabilidad de las clases populares puede ser explicada por procesos vinculados al poder social disponible, no solo personal, sino también de cariz colectivo. El contexto social que rodea a las personas que pertenecen a clases más desfavorecidas (vs. altas), viene caracterizado por la escasez de recursos y un menor poder social, así como por la presencia de un mayor número de estresores y fuentes potenciales de amenazas externas; en definitiva, por una mayor vulnerabilidad e incertidumbre que, a la postre, socavan la capacidad de eficacia y sensación de control respecto a las demandas del entorno (Kraus et al., 2012). En consonancia con lo anterior, se ha encontrado que los individuos de clases más bajas (vs. altas) tienden a mostrar menores niveles personales de eficacia percibida y sensación de control a lo largo de diferentes esferas (p.ej., Becker et al., 2017; Eom et al., 2018; Lachman \& Weaver, 1998). Por un lado, la evidencia sobre clase social y eficacia colectiva es relativamente limitada y, por otro, permite centrar la atención en aspectos de naturaleza comunitaria para así constatar si las personas de clases sociales más desfavorecidas manifiestan menor eficacia -su colectivismo podría amortiguar los efectos negativos del menor estatus y poder social (Hipp, 2016). Por último, también se ha indicado que una mayor eficacia colectiva se relaciona con resultados positivos a nivel de bienestar (p.ej., Bagci \& Canpolat, 2020).

\section{La presente investigación}

Este estudio pretende responder a los siguientes interrogantes enmarcados en el contexto de crisis pandémica a lo largo de varios países iberoamericanos (Argentina, Brasil, Chile, Colombia, España, México, Portugal y Perú): (a) ¿existen diferencias en bienestar y afectividad positiva y negativa en función de la clase social?; (b) ¿contribuye la clase social subjetiva a la predicción del bienestar y los estados afectivos positivo y negativo más allá de la influencia atribuida al género, la edad y la formación educativa?; y (c) ¿pueden las variables percepción de riesgo relativo a la crisis de la COVID-19 y la eficacia colectiva explicar -al menos parcialmente- los efectos de la clase social subjetiva en el bienestar y el afecto positivo y negativo?

\section{Figura 1}

Modelo de mediación con las variables riesgo percibido relativo a la COVID-19 y eficacia colectiva como mediadores de la relación entre clase social subjetiva y bienestar/afectividad

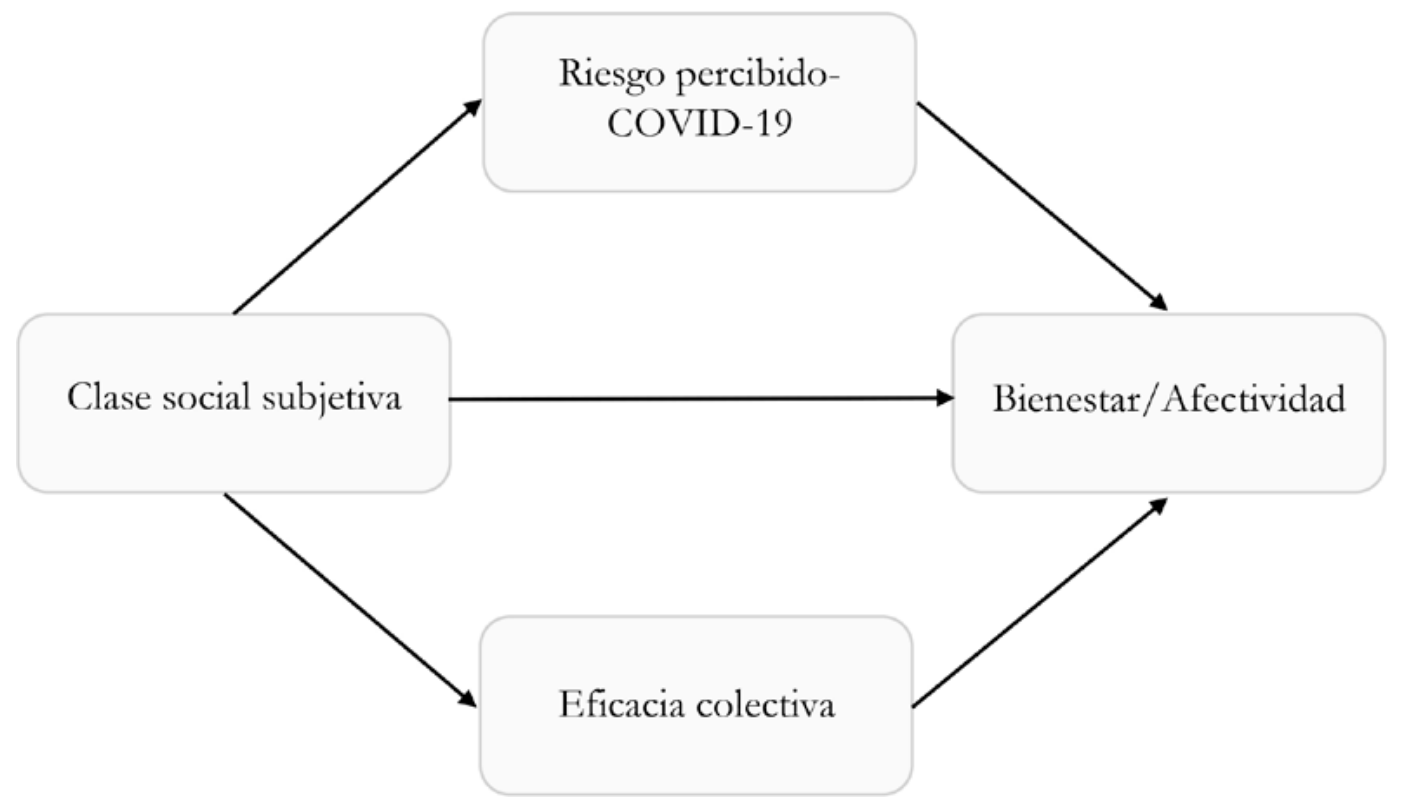

Nosotros proponemos que las personas que perciben pertenecer a una clase social más desfavorecida, en comparación con aquellas de clases más altas, presentarán menores niveles de bienestar y afecto positivo, por un lado, y un mayor afecto negativo, por otro. Asimismo, esperamos que la clase social subjetiva se revele como un predictor significativo de estos indicadores de bienestar y afectividad 
más allá del género, la edad y el nivel educativo. Por último, consideramos que los menores niveles de bienestar y afecto positivo (y mayores de afecto negativo) presentados por los individuos de clases sociales más bajas (vs. altas) podrían explicarse atendiendo a su mayor percepción de riesgo en relación con la pandemia y a su menor eficacia colectiva percibida a la hora de afrontar los efectos de la misma (Figura 1).

\section{Participantes y procedimiento}

\section{Método}

La presente investigación comprendió un total de 2113 participantes $(69.1 \%$ mujeres) con una edad media de 32.48 años $(D T=14.52)$. Los participantes, procedentes de un total de 8 países de lberoamérica (Argentina, Brasil, Chile, Colombia, España, México, Portugal y Perú), cumplimentaron una encuesta en línea durante los meses de abril y julio de 2020. Los participantes proporcionaron consentimiento informado y fueron informados de la confidencialidad de sus respuestas. Este trabajo se enmarca en un proyecto más amplio de corte transcultural, revisado y aprobado por el Comité de Ética de Investigación de la institución académica responsable. En esta investigación el foco se sitúa en países pertenecientes a la región cultural iberoamericana. El motivo radica en el fuerte vínculo histórico y cultural existente entre estos países. A diferencia de otras regiones (p.ej., la Commonwealth y la Francofonía), la iberoamericana es histórica y culturalmente homogénea y comparte, además, otra variedad de elementos vinculantes como señas de identidad y múltiples valores (Gratius, 2006). La Tabla 1 recoge de forma pormenorizada las características demográficas de cada una de las muestras.

Tabla 1

Descripción sociodemográfica de los participantes por país

\begin{tabular}{|c|c|c|c|c|c|c|}
\hline \multirow[t]{2}{*}{ País } & \multicolumn{4}{|c|}{ Edad (en años) } & \multicolumn{2}{|c|}{ Género $(n)$} \\
\hline & $N_{\text {total }}$ & $M(D T)$ & Rango & Mujer & Hombre & No binario/No responde \\
\hline Argentina & 138 & $26.53(12.25)$ & $19-73$ & 94 & 44 & 0 \\
\hline Brasil & 209 & $34.85(14.02)$ & $18-74$ & 137 & 71 & 1 \\
\hline Chile & 540 & $28.32(11.91)$ & $18-79$ & 363 & 166 & 11 \\
\hline Colombia & 329 & $29.83(15.66)$ & $18-77$ & 200 & 122 & 7 \\
\hline España & 538 & $35.65(13.93)$ & $18-90$ & 412 & 111 & 15 \\
\hline México & 169 & $27.63(10.38)$ & $18-60$ & 113 & 54 & 2 \\
\hline Perú & 55 & $42.85(17.92)$ & $18-78$ & 35 & 18 & 2 \\
\hline Portugal & 135 & $47.26(13.68)$ & $18-81$ & 107 & 27 & 1 \\
\hline Total & 2113 & $32.48(14.52)$ & $18-90$ & 1461 & 613 & 39 \\
\hline
\end{tabular}

\section{Instrumentos}

Bienestar personal. Evaluado a través de una selección de 6 ítems del General Health Questionnaire (GHQ-12; Goldberg, \& Williams, 1988; Rocha et al., 2011). La evaluación de cada una de las sentencias (p.ej., "¿Te sientes razonablemente feliz considerando todas las circunstancias?) siguió un formato tipo Likert con 4 opciones de respuesta comprendidas entre 0 (Nada) y 3 (Mucho). El índice de consistencia interna evaluado a través del coeficiente Alfa de Cronbach fue .83. Las puntuaciones de los ítems se codificaron de forma que, a mayor puntuación, mayor bienestar.

Afectividad positiva y negativa. El grado de afecto positivo (p.ej., ¿Cuán agradecido/a te has sentido?”) y negativo (p.ej.. "¿Cuán triste, desanimado/a o infeliz se ha sentido?") experimentados fue evaluado a través de la consideración de 11 ítems de la Escala de Emociones Positivas y Negativas de Fredrickson (2009). Se empleó un formato de respuesta tipo Likert de 5 puntos $(0=N a d a, 4=$ Mucho). Los coeficientes Alfa de Cronbach fueron .84 y .80 para afecto positivo y negativo, respectivamente.

Percepción de riesgo. Se administró una escala elaborada ad hoc para evaluar el nivel de riesgo percibido en relación con el contexto de pandemia de COVID-19. Se utilizaron un total de 7 ítems (p.ej., "Tengo miedo de enfermar del coronavirus"), con alternativa de respuesta múltiple de 1 (Completamente en desacuerdo) a 7 (Completamente de acuerdo). El coeficiente Alfa de Cronbach obtenido fue .61.

Eficacia colectiva. Se utilizó un instrumento creado ad hoc a fin de captar la percepción de capacidad de eficacia colectiva a la hora de abordar la situación derivada de la pandemia. Se trata de una prueba compuesta por un total de 3 ítems (p.ej.. "Sentimos que fuimos capaces de organizarnos para mejorar nuestra situación"), los cuales se respondían con un formato de respuesta tipo Likert de 7 puntos ( $1=$ Completamente en desacuerdo; 7 = Completamente de acuerdo). Los datos psicométricos obtenidos arrojaron un coeficiente Alfa de Cronbach de.76. 
Clase social. Se incluyó el nivel educativo como indicador de clase social objetiva. Dicho indicador comprendió un total de 7 categorías $(1=$ Menos de 6 años; $7=$ Doctorado). La clase social subjetiva fue evaluada a través de la Escala MacArthur de Estatus Socioeconómico Subjetivo (Adler et al., 2000). Bajo la forma de una representación gráfica de "escalera social" compuesta por 10 escalones, esta medida representa las diferentes posiciones socioeconómicas de los individuos en términos de ingresos, nivel educativo y ocupación. A fin de estimar la percepción subjetiva de clase social individual, los participantes seleccionan el escalón en el que se situarían en comparación con otras personas de la sociedad.

\section{Análisis estadísticos}

En primer lugar, calculamos los estadísticos descriptivos de las variables objeto de análisis en esta investigación. A continuación, estimamos los coeficientes de correlación del producto-momento (Pearson) entre dichas variables. Para interpretar la magnitud de los coeficientes de correlación, seguimos los criterios sugeridos por Richard et al. (2003), según los cuales, en el campo de la psicología social, correlaciones de $.10, .20$ y .30 corresponden a tamaños del efecto pequeño, mediano y grande, respectivamente. En un siguiente paso, llevamos a cabo una serie de análisis de regresión jerárquica (uno para cada variable criterio: bienestar, afectividad positiva y negativa) con el objeto de determinar la contribución específica de la clase social subjetiva a dichos indicadores de bienestar y afectividad más allá de la influencia atribuida a variables sociodemográficas clásicas (i.e., género y edad) y a la formación educativa. Así pues, en un primer paso incluimos las variables género, edad y nivel educativo y en un segundo paso añadimos la variable clase social subjetiva. Por último, examinamos el papel mediador de la percepción de riesgo relativa a la COVID-19 y la eficacia colectiva percibida en la relación entre clase social subjetiva y bienestar/afectividad positiva y negativa. Para ello, empleamos la macro PROCESS v3.4.1. para SPSS (modelo 4; Hayes, 2013), la cual permite estimar los efectos indirectos y sus correspondientes errores estándar e intervalos de confianza sobre la base del Bootstrap (i.e., remuestreo; en este caso se extrajeron 10000 muestras). Los efectos indirectos son considerados significativos cuando los intervalos de confianza construidos no contienen el valor 0 . Todos los análisis estadísticos fueron llevados a cabo a través del Statistical Package for the Social Sciences (SPSS) versión 25.0.

\section{Estadísticos descriptivos y correlaciones}

\section{Resultados}

La Tabla 2 recoge las medias y desviaciones típicas de las medidas evaluadas a lo largo de las diferentes muestras. Los correlatos del nivel educativo y la clase social subjetiva con el resto de variables analizadas para la muestra global aparecen en la Tabla 3.

Tabla 2

\begin{tabular}{|c|c|c|c|c|c|c|c|}
\hline País & Nivel educativo & $\begin{array}{l}\text { Clase social } \\
\text { subjetiva }\end{array}$ & Bienestar & $\begin{array}{l}\text { Afecto } \\
\text { Positivo }\end{array}$ & $\begin{array}{l}\text { Afecto } \\
\text { Negativo }\end{array}$ & $\begin{array}{l}\text { Percepción de } \\
\text { Riesgo }\end{array}$ & Eficacia colectiva \\
\hline & $M(D T)$ & $M(D T)$ & $M(D T)$ & $M(D T)$ & $M(D T)$ & $M(D T)$ & $M(D T)$ \\
\hline Argentina & $4.12(0.56)$ & $6.51(1.57)$ & $1.70(0.67)$ & $2.14(0.72)$ & $1.95(0.89)$ & $3.51(0.92)$ & $5.16(1.29)$ \\
\hline Brasil & $4.87(1.04)$ & $5.19(1.82)$ & $1.42(0.60)$ & $1.73(0.74)$ & $2.47(0.96)$ & $4.21(0.96)$ & $3.90(1.56)$ \\
\hline Chile & $4.32(0.81)$ & $4.92(1.53)$ & $1.29(0.63)$ & $1.94(0.72)$ & $2.17(0.89)$ & $4.23(1.02)$ & $4.43(1.33)$ \\
\hline Colombia & $4.37(0.81)$ & $6.61(1.53)$ & $1.59(0.62)$ & $2.40(0.77)$ & $1.90(0.95)$ & $3.77(0.95)$ & $4.84(1.49)$ \\
\hline España & $4.94(1.17)$ & $5.98(1.42)$ & $1.52(0.70)$ & $2.11(0.79)$ & $2.07(0.93)$ & $3.64(0.92)$ & $4.70(1.27)$ \\
\hline México & $4.21(0.90)$ & $5.81(1.54)$ & $1.50(0.58)$ & $2.03(0.72)$ & $1.83(0.91)$ & $4.00(1.14)$ & $4.20(1.45)$ \\
\hline Perú & $5.04(0.95)$ & $5.80(1.77)$ & $1.61(0.59)$ & $2.16(0.71)$ & $1.87(0.93)$ & $4.13(1.14)$ & $4.63(1.43)$ \\
\hline Portugal & $4.87(1.20)$ & $5.89(1.61)$ & $1.72(0.66)$ & $1.92(0.87)$ & $1.73(0.98)$ & $3.68(1.04)$ & $4.77(1.22)$ \\
\hline Total & $4.57(1.01)$ & $5.74(1.66)$ & $1.49(0.66)$ & $2.06(0.78)$ & $2.05(0.95)$ & $3.90(1.02)$ & $4.57(1.40)$ \\
\hline
\end{tabular}

A lo largo de la muestra general, tal y como se puede constatar en la Tabla 3, la clase social subjetiva correlacionó moderada y positivamente con las medidas de bienestar $(r=.25, p<.001)$ y afecto positivo $(r=.19, p<.001)$. Asimismo, una menor clase social subjetiva se relacionó con mayores niveles de afectividad negativa $(r=-.16, p<.001)$. Los datos obtenidos también arrojaron, por un lado, una correlación negativa moderada entre clase social subjetiva y percepción de riesgo vinculada a la crisis de la COVID-19 $(r=-.22, p<.001)$ y, por otro, una correlación positiva de moderada a alta entre clase social subjetiva y eficacia colectiva percibida $(r=.28, p<.001)$. Tal y como también muestra la Tabla 3, la variable nivel educativo mostró el mismo patrón de correlaciones que la clase subjetiva, aunque sus coeficientes de correlación fueron siempre más bajos, reflejando tamaños del efecto de bajos a moderados. 
GINÉS NAVARRO-CARRILLO, LANDER MÉNDEZ, HUSEYIN CAKAL, DARÍO PÁEZ, JOSÉ J. PIZARRO, LARRAITZ ZUMETA, MARCELA GRACIA-LEIVA SILVIA DA COSTA NEKANE BASABE SAIOA TELLETXEA GISELA DELFINO, ELZA TECHIO, ANDERSON MATHIAS, CAROLINAALZUGARAY-PONCE, LORETO VILLAGRÁN, GONZALO MARTÍNEZ-ZELAYA, MARIAN BILBAO, ANNA WLODARCZYK, WILSON LÓPEZ-LÓPEZ, LAURA ALFARO-BERACOECHEA, MANUEL. L. IBARRA, CATARINA CARVALHO, ISABEL. R. PINTO, EMILIO MOYANO-DÍAZ, AGUSTÍN ESPINOSA, ROSA. M. CUETO

Tabla 3

Correlaciones bivariadas entre las variables de interés en toda la muestra

\begin{tabular}{|c|c|c|c|c|c|c|c|}
\hline Variables & 1 & 2 & 3 & 4 & 5 & 6 & 7 \\
\hline Nivel educativo & --- & & & & & & \\
\hline Clase social subjetiva & $.17^{\star \star \star}$ & --- & & & & & \\
\hline Percepción de riesgo & $-.10^{\star \star *}$ & $-.22^{* \star *}$ & --- & & & & \\
\hline Eficacia colectiva & $.05^{*}$ & $.28^{* \star *}$ & $-.13^{\star \star *}$ & -- & & & \\
\hline Bienestar & $.17^{\star \star \star}$ & $.25^{\star \star \star}$ & $-.41^{\star \star *}$ & $.25^{\star \star \star}$ & --- & & \\
\hline Afecto positivo & $.05^{*}$ & $.19^{\star \star \star}$ & $-.18^{\star \star \star}$ & $.28^{* \star *}$ & $.49^{\star \star \star}$ & --- & \\
\hline Afecto negativo & $-.09^{\star * *}$ & $-.16^{\star * *}$ & $.41^{\star \star \star}$ & $-.18^{\star \star *}$ & $-.66^{* \star \star}$ & $-.31^{* \star *}$ & --- \\
\hline
\end{tabular}

Nota: ${ }^{*} p<.05 ;{ }^{* \star} p<.01 ;{ }^{* \star} p<.001$.

En lo tocante a los correlatos del nivel educativo y la clase social subjetiva con el resto de variables a nivel de país (Tabla 4), nuestros resultados revelaron, en general, que el tamaño de las asociaciones entre la clase social subjetiva y el resto de variables fue superior al de las relaciones del nivel educativo. Asimismo, se observó una correlación positiva entre clase social subjetiva y bienestar en todos los países considerados (a excepción de Colombia y México); la relación más alta se obtuvo en la muestra de Portugal $(r=.36, p<.001)$. Las correlaciones entre la clase social subjetiva y los indicadores de afectividad fueron más bajas. Las relaciones numéricamente más altas se obtuvieron en la muestra española (en el caso del afecto positivo: $r=.18, p<.001$ ) y en la portuguesa (en el caso del afecto negativo: $r=-.18, p=.038$ ), siendo ambas de bajas a moderadas. Tal y como también se aprecia en la Tabla 4, las muestras correspondientes a Portugal $(r=-.24, p=.005)$ y España $(r=-.22$, $p<.001)$ mostraron las correlaciones negativas numéricamente más elevadas entre clase social subjetiva y riesgo percibido, las cuales reflejan un tamaño del efecto mediano. Por último, las muestras de Argentina $(r=.33, p<.001)$ y Brasil $(r=.30, p<.001)$ presentaron correlaciones positivas altas entre clase social subjetiva y eficacia colectiva percibida.

Tabla 4

Correlaciones bivariadas entre nivel educativo y clase social subjetiva y resto de variables de interés por país

\begin{tabular}{|c|c|c|}
\hline & Nivel educativo & Clase social subjetiva \\
\hline \multicolumn{3}{|l|}{ Argentina } \\
\hline Bienestar & .09 & $.17^{*}$ \\
\hline Afecto positivo & -.07 & .08 \\
\hline Afecto negativo & -.08 & -.03 \\
\hline Percepción de riesgo & .06 & $-.19^{*}$ \\
\hline Eficacia colectiva & -.03 & $.33^{* * *}$ \\
\hline \multicolumn{3}{|l|}{ Brasil } \\
\hline Bienestar & .09 & $.26^{\star \star *}$ \\
\hline Afecto positivo & .07 & .06 \\
\hline Afecto negativo & -.11 & $-.13^{\dagger}$ \\
\hline Percepción de riesgo & $-.15^{*}$ & -.09 \\
\hline Eficacia colectiva & .10 & $.30^{\star \star \star}$ \\
\hline \multicolumn{3}{|l|}{ Chile } \\
\hline Bienestar & $.20^{* * *}$ & $.22^{* \star *}$ \\
\hline Afecto positivo & $.15^{\star * *}$ & $.14^{* *}$ \\
\hline Afecto negativo & $-.12^{* *}$ & $-.16^{* * *}$ \\
\hline Percepción de riesgo & $-.08^{\dagger}$ & $-.19^{\star \star \star}$ \\
\hline Eficacia colectiva & .02 & $.27^{\star \star *}$ \\
\hline \multicolumn{3}{|l|}{ Colombia } \\
\hline Bienestar & $.30^{* * *}$ & .06 \\
\hline Afecto positivo & $.18^{* *}$ & $.10^{\dagger}$ \\
\hline Afecto negativo & $-.23^{* * *}$ & -.02 \\
\hline Percepción de riesgo & $-.11^{*}$ & $-.11^{*}$ \\
\hline Eficacia colectiva & $.10^{\dagger}$ & $.24^{* * *}$ \\
\hline \multicolumn{3}{|l|}{ España } \\
\hline Bienestar & $.17^{* * *}$ & $.24^{* * *}$ \\
\hline Afecto positivo & -.01 & $.18^{\star * *}$ \\
\hline Afecto negativo & $-.11^{*}$ & $-.17^{\star * *}$ \\
\hline Percepción de riesgo & -.07 & $-.22^{* * *}$ \\
\hline Eficacia colectiva & .05 & $.25^{\star \star \star}$ \\
\hline
\end{tabular}




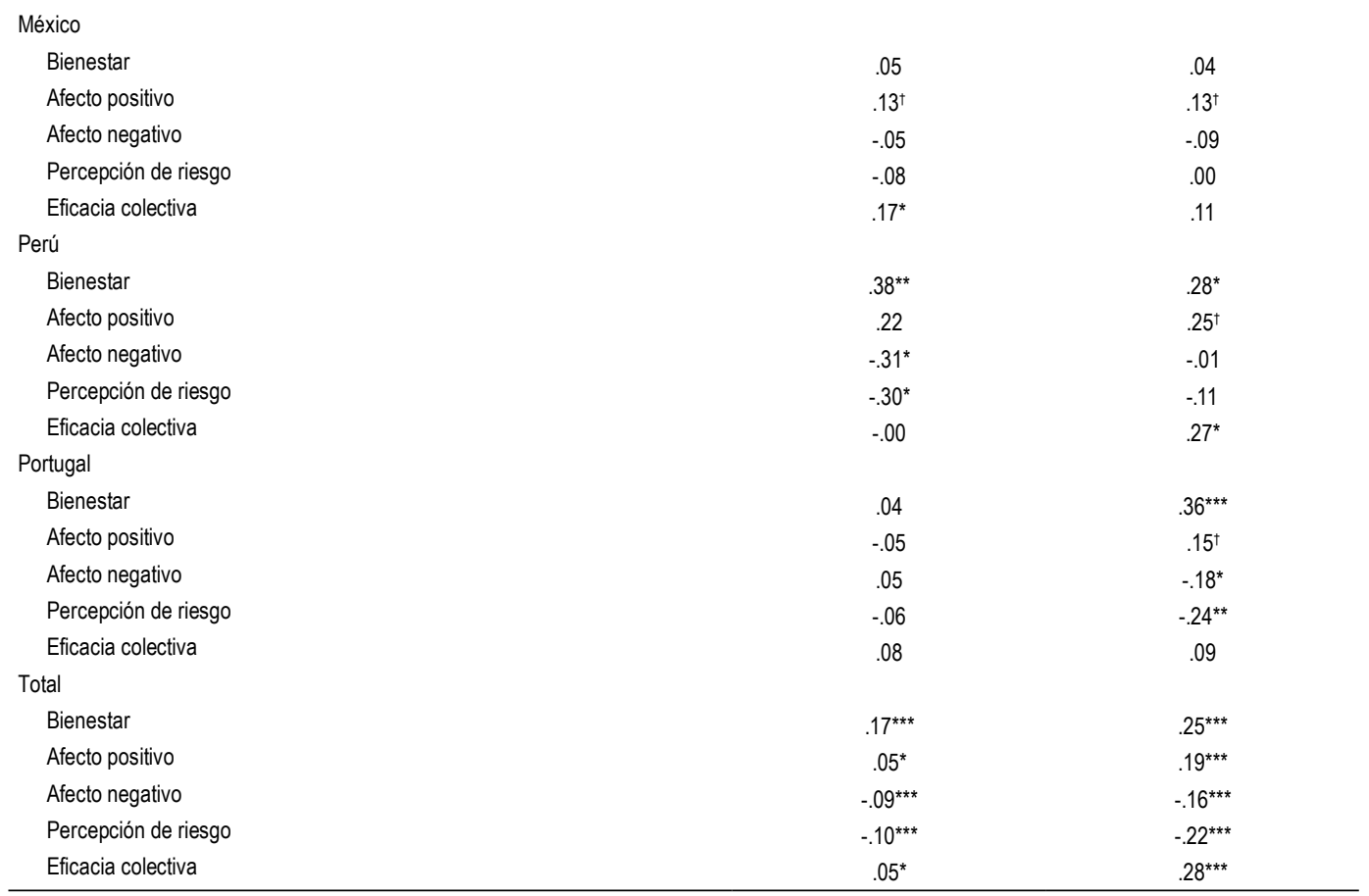

Nota: $\dagger p \leq .10 ;{ }^{*} p<.05 ;{ }^{* *} p<.01 ;{ }^{* * *} p<.001$.

\section{Análisis de regresión jerárquica}

Los resultados de los análisis de regresión jerárquica dirigidos a examinar la contribución de la clase social subjetiva al bienestar, afecto positivo y negativo más allá del género, la edad y el nivel educativo ${ }^{1}$ aparecen en la Tabla 5.

\section{Bienestar}

Los resultados obtenidos revelaron que tanto el género, la edad como el nivel educativo se relacionaron significativamente con la variable bienestar; en concreto, el hecho de ser mujer $(\beta=-.18, p<.001)$, tener menor edad $(\beta=.29, p<.001)$ y contar con una formación educativa más baja $(\beta=.05, p=.018)$ se vinculaba con un menor bienestar.

La variable clase social subjetiva, introducida en el siguiente paso, se asoció positiva y significativamente al bienestar $(\beta=.20, p<.001)$ más allá del género, la edad y el nivel educativo. Cabe señalar además que la incorporación de esta variable a la ecuación de regresión explicó un $4 \%$ adicional de la varianza en bienestar, siendo este incremento estadísticamente significativo $\Delta F(1,2063)=99.71, p<$ .001 .

\section{Afecto positivo}

Los resultados mostraron, al igual que en el caso de la variable criterio precedente, que ser mujer $(\beta=$ $-.05, p=.024)$ y tener menor edad $(\beta=.18, p<.001)$ fue indicativo de menores niveles de afectividad positiva. El nivel educativo no se relacionó significativamente con el afecto positivo $(\beta=-.03, p=.288$ ).

La clase social subjetiva, en consonancia con lo hallado en relación con el bienestar, también se asoció significativamente con el afecto positivo. Una menor clase social subjetiva fue indicativa de menores puntuaciones en afectividad positiva $(\beta=.18, p<.001)$. Este efecto se dio aun controlando el género, la edad y el nivel educativo. Además, la inclusión de la clase social subjetiva en el segundo paso de la ecuación de regresión explicó un 3.1\% adicional de la varianza en afectividad positiva; dicho incremento fue estadísticamente significativo, $\Delta F(1,2063)=68.77, p<.001$.

\section{Afecto negativo}

Los resultados evidenciaron un patrón similar al hallado en el caso del afecto positivo. Concretamente, ser mujer $(\beta=.16, p<.001)$ y ser más joven $(\beta=-.27, p<.001)$ fue indicativo de mayores niveles de afectividad negativa. El nivel educativo no se asoció significativamente a esta variable criterio $(\beta=.02$, $p=.439$ ).

Una menor clase social subjetiva se conectó con una mayor inclinación a presentar afectividad negativa $(\beta=-.13, p<.001)$ más allá de los efectos atribuidos al género, la edad y el nivel educativo. Al igual que en los casos anteriores, la inclusión de la clase social subjetiva en el segundo paso de

\footnotetext{
1 De forma preliminar, dada la posible estructura jerárquica de los datos, llevamos a cabo una aproximación multinivel para conocer el porcentaje de variabilidad de las variables criterio (i.e., bienestar, afectividad positiva y negativa) que explicaba el factor país; dado que dicha variabilidad fue exigua en el caso de todas las variables criterio $(\leq 6 \%$; un $10 \%$ es considerado bajo [(Kahn, 2011)], realizamos un análisis de regresión con toda la muestra.
} 
GINÉS NAVARRO-CARRILLO, LANDER MÉNDEZ, HUSEYIN CAKAL, DARÍO PÁEZ, JOSÉ J. PIZARRO, LARRAITZ ZUMETA, MARCELA GRACIA-LEIVA, SILVIA DA COSTA, NEKANE BASABE, SAIOA TELLETXEA, GISELA DELFINO, ELZA TECHIO, ANDERSON MATHIAS, CAROLINA ALZUGARAY-PONCE, LORETO VILLAGRÁN, GONZALO MARTÍNEZ-ZELAYA, MARIAN BILBAO, ANNA WLODARCZYK, WILSON LÓPEZ-LÓPEZ, LAURA ALFARO-BERACOECHEA, MANUEL. L. IBARRA, CATARINA CARVALHO, ISABEL. R. PINTO, EMILIO MOYANO-DÍAZ, AGUSTÍN ESPINOSA, ROSA. M. CUETO

la ecuación de regresión explicó un incremento significativo (1.5\%) en la varianza en afecto negativo, $\Delta F(1,2063)=35.40, p<.001$.

Tabla 5

Análisis de regresión jerárquica con variables sociodemográficas (i.e., género y edad), nivel educativo y clase social subjetiva como predictores del bienestar y la afectividad

\begin{tabular}{|c|c|c|c|c|c|c|c|c|c|c|c|c|}
\hline \multirow[t]{2}{*}{ Predictores } & \multicolumn{4}{|c|}{ Bienestar } & \multicolumn{4}{|c|}{ Afecto positivo } & \multicolumn{4}{|c|}{ Afecto negativo } \\
\hline & $\beta$ & $t$ & $P$ & $R^{2}\left(\Delta R^{2}\right)$ & $\beta$ & $t$ & $p$ & $R^{2}\left(\Delta R^{2}\right)$ & $\beta$ & $t$ & $p$ & $R^{2}\left(\Delta R^{2}\right)$ \\
\hline Paso 1 & & & & $.13\left(.13^{\star \star *}\right)$ & & & & $.03\left(.03^{\star * \star}\right)$ & & & & $.10\left(.10^{\star * *}\right)$ \\
\hline Género & -.18 & -8.72 & $<.001$ & & -.05 & -2.26 & .024 & & .16 & 7.86 & $<.001$ & \\
\hline Edad & .29 & 12.84 & $<.001$ & & .18 & 7.74 & $<.001$ & & -.27 & -12.08 & $<.001$ & \\
\hline Nivel educativo & .05 & 2.36 & .018 & & -.03 & -1.06 & .288 & & .02 & 0.77 & .439 & \\
\hline Paso 2 & & & & $.17\left(.04^{\star \star \star}\right)$ & & & & $.06\left(.03^{\star * *}\right)$ & & & & $.12\left(.02^{\star \star *}\right)$ \\
\hline Género & -.16 & -8.04 & $<.001$ & & -.03 & -1.58 & .114 & & .15 & 7.39 & $<.001$ & \\
\hline Edad & .28 & 12.92 & $<.001$ & & .18 & 7.68 & $<.001$ & & -.27 & -12.04 & $<.001$ & \\
\hline Nivel educativo & .02 & 0.92 & .359 & & -.05 & -2.29 & .022 & & .04 & 1.65 & .100 & \\
\hline Clase social subjetiva & .20 & 9.99 & $<.001$ & & .18 & 8.29 & $<.001$ & & -.13 & -5.95 & $<.001$ & \\
\hline
\end{tabular}

\section{El rol mediador del riesgo percibido y la eficacia colectiva}

Una vez examinada la contribución específica de la clase social subjetiva al bienestar y la afectividad general, pasamos a comprobar si el riesgo percibido relativo a la COVID-19 y la eficacia colectiva mediaban de forma simultánea la relación entre clase social subjetiva y bienestar/afectividad (positiva y negativa).

Clase social subjetiva, riesgo percibido, eficacia colectiva y bienestar

Los resultados mostraron que todos los path fueron significativos: una menor clase social subjetiva se relacionó con una mayor percepción de riesgo $(b=-.14, E S=.01, p<.001)$, una menor eficacia colectiva $(b=.23, E S=.02, p<.001)$ y menores niveles de bienestar $(b=.10, E S=.01, p<.001)$. Una mayor percepción de riesgo $(b=-.24, E S=.01, p<.001)$ y una menor eficacia colectiva $(b=.08, E S=$

$.01, p<.001)$ se relacionaron con menores niveles de bienestar. Los efectos indirectos de percepción de riesgo, $E I=.03, E S=.004(95 \%$ IC $[.025, .039])$, y eficacia colectiva, $E I=.02, E S=.003$ (95\% IC $[.013, .024]$, fueron significativos, toda vez que los intervalos de confianza generados no contienen el valor 0 . Una vez incluidas en el modelo las variables mediadoras (i.e., percepción de riesgo y eficacia colectiva), el efecto de la clase social subjetiva en el bienestar siguió siendo significativo $(b=.05, E S=$ $.01, p<.001)$, indicando la existencia de una mediación parcial.

Clase social subjetiva, riesgo percibido, eficacia colectiva y afecto positivo

Los datos obtenidos indicaron que todos los path fueron significativos: una menor clase social subjetiva se relacionó con una mayor percepción de riesgo $(b=-.14, E S=.01, p<.001)$, una menor eficacia colectiva $(b=.23, E S=.02, p<.001)$ y un menor afecto positivo $(b=.09, E S=.01, p<.001)$. Una mayor percepción de riesgo $(b=-.10, E S=.02, p<.001)$ y una menor eficacia colectiva $(b=.13, E S=$ $.01, p<.001)$ se relacionaron con una menor inclinación a manifestar afectividad positiva. Al igual que en el caso del bienestar, los efectos indirectos de percepción de riesgo, El = .01, ES =.003 (95\% IC $[.008, .018])$, y eficacia colectiva, $E I=.03, E S=.004$ (95\% IC [.024, .039], fueron significativos, pues los intervalos de confianza generados no comprenden el valor 0 . Una vez consideradas en el modelo las variables mediadoras, el efecto de la clase social subjetiva en la afectividad positiva siguió siendo significativo $(b=.05, E S=.01, p<.001)$. Esto refleja la existencia de una mediación parcial.

Clase social subjetiva, riesgo percibido, eficacia colectiva y afecto negativo

Los datos obtenidos mostraron nuevamente que todos los path fueron significativos: una menor clase social subjetiva se relacionó con una mayor percepción de riesgo $(b=-.14, E S=.01, p<.001)$, una menor eficacia colectiva $(b=.23, E S=.02, p<.001)$ y mayores niveles de afectividad negativa $(b=$ $-.09, E S=.01, p<.001)$. Una mayor percepción de riesgo $(b=.36, E S=.02, p<.001)$ y una menor eficacia colectiva $(b=-.08, E S=.01, p<.001)$ se relacionaron con una mayor afectividad negativa. En consonancia con los casos precedentes, los efectos indirectos de percepción de riesgo, $E I=-.05, E S$ $=.005(95 \%$ IC [-.059, -.038]), y eficacia colectiva, $E I=-.02, E S=.004$ (95\% IC [-.026, -.011], fueron significativos, habida cuenta de que el 0 no se encontraba incluido en los intervalos de confianza. Cuando se incluyeron en el modelo las variables mediadoras, el efecto de la clase social subjetiva en 
la afectividad negativa dejó de ser significativo $(b=-.02, E S=.01, p=.061)$, lo que indica la existencia de una mediación completa.

En síntesis, los datos obtenidos mostraron que una menor clase social subjetiva se relacionó con un menor bienestar, un menor afecto positivo y un mayor afecto negativo de manera indirecta a través de mayores niveles de riesgo percibido y menores de eficacia colectiva.

\section{Discusión}

Esta investigación perseguía poner a prueba la relación entre la clase social y bienestar y afectividad (positiva y negativa) durante la pandemia de COVID-19. Más específicamente, estábamos interesados en (a) analizar la contribución específica de la clase social subjetiva al bienestar y afectividad general más allá del nivel educativo y (b) determinar si la percepción de riesgo relacionada con la COVID-19 y la eficacia colectiva percibida median simultáneamente los efectos de la clase social subjetiva en el bienestar y en el afecto positivo y negativo. Hasta donde llega nuestro conocimiento, este es el primer trabajo empírico focalizado en el análisis sistemático del papel de la clase social subjetiva (vs. nivel educativo) en relación con el bienestar y el afecto (y de potenciales mecanismos psicosociales subyacentes) durante la pandemia.

Nuestros resultados confirmaron la existencia de diferencias en bienestar y afectividad positiva y negativa en los participantes en función de su clase social. Resulta de gran importancia mencionar que la clase social subjetiva se relacionó positivamente tanto con el bienestar como con los dos indi- cadores de afectividad aun controlando la edad, el género y el nivel educativo. El nivel educativo solo se asoció significativamente a la medida de bienestar. No obstante, su coeficiente estandarizado de regresión ( $\beta$ $=.05)$ fue más bajo en comparación con el de la clase social subjetiva $(\beta=.20)$. Así pues, estos datos confirman, en el contexto actual de crisis pandémica, (a) que la clase social subjetiva constituye, en comparación con el nivel educativo, un mejor predictor del bienestar, replicando así diferentes investigaciones previas (p.ej., Cundiff \& Matthews, 2017; Navarro-Carrillo et al., 2020; Tan et al., 2020); y (b), por tanto, la necesidad de que estudios futuros presten mayor atención a la clase social de los individuos cuando analicen el impacto psicosocial de la pandemia de COVID-19. Asimismo, este estudio contribuye a complementar investigaciones recientes que analizaron únicamente el impacto de facetas objetivas de clase social en el bienestar (Wanberg et al., 2020).

Esta investigación también contribuye a avanzar en la comprensión de los procesos psicosociales susceptibles de explicar la asociación entre la clase social y el bienestar/afectividad, aspecto que representa uno de los principales retos en la literatura sobre clase social y bienestar (Diener et al., 2010). Esta investigación sugiere que, en el escenario de crisis pandémica, una posible vía a través de la cual la clase social subjetiva se relaciona con todos los indicadores de bienestar y afectividad evalua- dos viene dada por la acción de las variables riesgo percibido relativo a la COVID-19 y percepción de eficacia colectiva. Más específicamente, los participantes de menor rango social percibido estaban más inclinados a sentirse en riesgo debido a las potenciales consecuencias de la pandemia en su vida (p.ej., a nivel de salud o económico) y a percibir menor eficacia colectiva a la hora de enfrentar el impacto de la misma, asociándose a su vez esta mayor percepción de riesgo y menor eficacia colectiva a menores niveles de bienestar y afecto positivo, por un lado, y mayores de afecto negativo, por otro. Estos hallazgos ayudan a ampliar el conocimiento disponible acerca de cómo se conecta la clase social subjetiva con el bienestar y la afectividad, en este caso durante la pandemia de COVID-19.

\section{Limitaciones y direcciones futuras de investigación}

Aunque esta investigación permite ahondar en uno de los ámbitos de investigación que mayor atención ha suscitado en las últimas décadas en la literatura psicológica sobre clase social, conviene mencionar sucintamente algunas de sus limitaciones. Por un lado, el procedimiento de muestreo no probabilístico impide la generalización de resultados. Por otro, la utilización de metodología no experimental no nos permite establecer inferencias de causalidad en la relación entre clase social subjetiva y bienestar/ afectividad. Conviene señalar también que el constructo riesgo percibido en el marco de la COVID-19 fue evaluado con una medida elaborada ad-hoc que presentó un coeficiente alfa de Cronbach relativamente bajo $(\alpha=.61)$

Futuros trabajos deberían -además de aspirar a la utilización de métodos de muestreo probabilístico- considerar realizar investigaciones longitudinales al objeto de dilucidar la posible relación de causalidad entre la percepción subjetiva de clase social y el bienestar/afectividad. Asimismo, existen trabajos recientes que han propuesto una fórmula alternativa de evaluación de la clase social subjetiva; por ejemplo, a través de una adaptación de la Escala MacArthur de Estatus Socioeconómico Subjetivo (Navarro-Carrillo et al., 2020), consistente en la administración de una escalera social para cada uno 
de los componentes de clase recogidos en la medida tradicional (i.e., ingresos, educación y ocupación). Dado que esta aproximación permite predecir varianza específica en bienestar no recogida en la Escala MacArthur de Estatus Socioeconómico Subjetivo, investigaciones futuras podrían considerar esta nueva forma de abordar la medición de la clase social subjetiva.

\section{Conclusiones}

Este trabajo arroja resultados que, a lo largo de varios países del área cultural latino-europea, confirman la contribución específica de la clase social subjetiva al bienestar y afectividad positiva y negativa en un contexto global de excepcionalidad, como lo es el representado por la pandemia de COVID-19. El presente estudio también confirma el rol mediador del riesgo percibido y la eficacia colectiva en la relación clase social subjetiva-bienestar/afectividad, contribuyendo así a esclarecer algunos de sus posibles mecanismos psicosociales explicativos. Los hallazgos discutidos confirman la relevancia de la clase social subjetiva en el estudio del impacto psicosocial de la COVID-19 en el bienestar y la afectividad.

\section{Agradecimientos:}

Esta investigación ha sido posible gracias a los Gobiernos de España (PID2020-115738GB-I0; PSI201784145-P; PSI2017-83966-R) y País Vasco (IT1187-19; PRE_2017_1_0405), la Universidad del País Vasco (DOCREC20/23), CONICYT (72180394) y la Universidad Viña del Mar (FIIUVM-EN-1904).

Los autores del manuscrito declaran la ausencia de conflicto de intereses.

\section{Referencias}

1. Adler, N. E., Epel, E. S., Castellazzo, G., \& Ickovics, J. R. (2000). Relationship of subjective and objective social status with psychological and physiological functioning: Preliminary data in healthy white women. Health Psychology, 19, 586-592. https://doi.org/10.1037/0278-6133.19.6.586

2. Alonso-Ferres, M., Navarro-Carrillo, G., Garrido-Macías, M., Moreno-Bella, E., \& Valor-Segura, I. (2020). Connecting perceived economic threat and prosocial tendencies: The explanatory role of empathic concern. Plos One, 15, e0232608. https://doi.org/10.1371/journal.pone.0232608

3. Afridi, L., \& Block, L. (2020). Frontline communities hit hardest by COVID-19. Association for Neighborhood \& Housing Development. Recuperado de: https://anhd.org/blog/frontlinecommunities-hit-hardest-COVID-19

4. Bagci, S. C., \& Canpolat, E. (2020). Group efficacy as a moderator on the associations between perceived discrimination, acculturation orientations, and psychological well-being. Journal of Community \& Applied Social Psychology, 30, 45-58. https://doi.org/10.1002/casp.2421

5. Becker, J. C., Kraus, M. W., \& Rheinschmidt-Same, M. (2017). Cultural expressions of social class and their implications for ingroup-related beliefs and behaviors. Journal of Social Issues, 73, 158-174. https://doi.org/10.1111/josi.12209

6. Blow, C. M. (2020). Social distancing is a privilege: The idea that this virus is an equal-opportunity killer must itself be killed. The New York Times. Recuperado de: https://www.nytimes. com/2020/04/05/opinion/coronavirus-social-distancing.html

7. Boyce, C. J., Brown, G. D. A., \& Moore, S. C. (2010). Money and happiness: Rank of income, not income, affects life satisfaction. Psychological Science, 21, 471-475. https://doi. org/10.1177/0956797610362671

8. Boyce, C. J., Delaney, L., \& Wood, A. M. (2018). The Great Recession and subjective well-being: How did the life satisfaction of people living in the United Kingdom change following the financial crisis? Plos One, 13(8), e0201215. https://doi.org/10.1371/journal.pone.0201215

9. Cundiff, J. M., \& Matthews, K. A. (2017). Is subjective social status a unique correlate of physical health? A meta-analysis. Health Psychology, 36, 1109-1125. https://doi.org/10.1037/hea0000534

10. Diener, E., Ng, W., Harter, J., \& Arora, R. (2010). Wealth and happiness across the world: material prosperity predicts life evaluation, whereas psychosocial prosperity predicts positive feeling. Journal of Personality and Social Psychology, 99, 52-61. https://doi.org/10.1037/a0018066

11. Diener, E., \& Oishi, S. (2000). Money and happiness: Income and subjective well-being across nations. En E.Diener \& E. M.Suh (Eds.), Culture and subjective well-being (pp. 185-218). Cambridge, MA: The MIT Press.

12. Diener, E., Oishi, S., \& Lucas, R. E. (2003). Personality, culture, and subjective well-being: Emotional and cognitive evaluations of life. Annual Review of Psychology, 54, 403-425. https://doi. org/10.1146/annurev.psych.54.101601.145056 
13. Eom, K., Kim, H. S., \& Sherman, D. K. (2018). Social class, control, and action: Socioeconomic status differences in antecedents of support for pro-environmental action. Journal of Experimental Social Psychology, 77, 60-75. https://doi.org/10.1016/j.jesp.2018.03.009

14. Fredrickson, B. L. (2009). Positivity. New York: Three Rivers Press.

15. Goldberg, D.P., \& Williams P. (1988). A user's guide to the General Health Questionnaire. UK: NFER-NELSON.

16. Gratius, S. (2014). La Cumbre Iberoamericana de Salamanca: ¿de una comunidad cultural a una alianza política? IBEROAMERICANA. América Latina - España - Portugal, 6, 171-176. https:// doi.org/10.18441/ibam.6.2006.21.171-176.

17. Han, Q., Zheng, B., Agostini, M., Belanger, J. J., Gutzkow, B., Kreienkamp, J., ... \& Leander, N. P. (2021). Associations of risk perception of COVID-19 with emotion and mental health during the pandemic. Journal of Affective Disorders. https://doi.org/10.1016/j.jad.2021.01.049

18. Hayes, A. F. (2013). Introduction to mediation, moderation, and conditional process analysis: $A$ regression-based approach. New York, NY: The Guilford Press.

19. Hipp, J. R. (2016). Collective efficacy: How is it conceptualized, how is it measured, and does it really matter for understanding perceived neighborhood crime and disorder? Journal of Criminal Justice, 46, 32-44. https://doi.org/10.1016/j.jcrimjus.2016.02.016

20. Kahn, J. H. 2011. Multilevel modeling: Overview and applications to research in counseling psychology. Journal of Counseling Psychology, 58, 257-271.

21. Kraus, M. W., Piff, P., \& Keltner, D. (2009). Social class, sense of control, and social explanation. Journal of Personality and Social Psychology, 97, 992-1004. https://doi.org/10.1037/a0016357

22. Kraus, M. W., Piff, P. K., Mendoza-Denton, R., Rheinschmidt, M. L., \& Keltner, D. (2012). Social class, solipsism, and contextualism: How the rich are different from the poor. Psychological Review, 119, 546-572. https://doi.org/10.1037/a0028756

23. Lachman, M. E., \& Weaver, S. L. (1998). The sense of control as a moderator of social class differences in health and well-being. Journal of Personality and Social Psychology, 74, 763-773. http://dx.doi.org/10.1037/0022-3514.74.3.763

24. Manstead, A. S. (2018). The psychology of social class: How socioeconomic status impacts thought, feelings, and behaviour. British Journal of Social Psychology, 57, 267-291. https://doi. org/10.1111/bjso.12251

25. Moya, M., \& Fiske, S. T. (2017). The social psychology of the great recession and social class divides. Journal of Social Issues, 73, 8-22. https://doi.org/10.1111/josi.12201

26. Navarro-Carrillo, G., Alonso-Ferres, M., Moya, M., \& Valor-Segura, I. (2020). Socioeconomic status and psychological well-being: Revisiting the role of subjective socioeconomic status. Frontiers in Psychology, 11, 1303. https://doi.org/10.3389/fpsyg.2020.01303

27. Navarro-Carrillo, G., Valor-Segura, I., \& Moya, M. (2019). The consequences of the perceived impact of the Spanish economic crisis on subjective well-being: The explanatory role of personal uncertainty. Current Psychology. https://doi.org/10.1007/s12144-019-00506-4

28. Organización Mundial de la Salud (OMS) (2020). Recuperado de: https://www.who.int/ directorgeneral/speeches/detail/who-director-general-s-opening-remarks-at-the-media-briefing-oncovid-19 11-march-2020 (31 de enero, 2021).

29. Pons-Valls, E. (2020). Covid-19, un virus que sí entiende de clases sociales, como todos. Público. Recuperado de: https://www.publico.es/sociedad/coronavirus-covid-19-virus-entiende-clases-sociales.html

30. Richard, F. D., Bond, C. F., Jr., \& Stokes-Zoota, J. J. (2003). One hundred years of social psychology quantitatively described. Review of General Psychology, 7, 331-363. https://doi. org/10.1037/1089-2680.7.4.331

31. Rocha, K. B., Pérez, K., Rodríguez-Sanz, M., Borrell, C., \& Obiols, J. E. (2011). Propiedades psicométricas y valores normativos del General Health Questionnaire (GHQ-12) en población general española. International Journal of Clinical and Health Psychology, 11, 125-139.

32. Rodríguez-Bailón, R. (2020). Inequality viewed through the mirror of COVID-19 (La desigualdad ante el espejo del COVID-19). International Journal of Social Psychology, 35, 647-655. https:// doi.org/10.1080/02134748.2020.1796298

33. Snibbe, A. C., \& Markus, H. R. (2005). You can't always get what you want: educational attainment, agency, and choice. Journal of Personality and Social Psychology, 88, 703-720. https://doi. org/10.1037/0022-3514.88.4.703 
GINÉS NAVARRO-CARRILLO, LANDER MÉNDEZ, HUSEYIN CAKAL, DARÍO PÁEZ, JOSÉ J. PIZARRO, LARRAITZ ZUMETA,

MARCELA GRACIA-LEIVA, SILVIA DA COSTA, NEKANE BASABE, SAIOA TELLETXEA, GISELA DELFINO, ELZA TECHIO,

ANDERSON MATHIAS, CAROLINAALZUGARAY-PONCE, LORETO VILLAGRÁN, GONZALO MARTÍNEZ-ZELAYA, MARIAN BILBAO,

ANNA WLODARCZYK, WILSON LÓPEZ-LÓPEZ, LAURA ALFARO-BERACOECHEA, MANUEL. L. IBARRA, CATARINA CARVALHO, ISABEL. R. PINTO, EMILIO MOYANO-DÍAZ, AGUSTÍN ESPINOSA, ROSA. M. CUETO

34. Tan, J. J. X., Kraus, M. W., Carpenter, N. C., \& Adler, N. E. (2020). The association between objective and subjective socioeconomic status and subjective well-being: A meta-analytic review. Psychological Bulletin, 146, 970-1020. https://doi.org/10.1037/bul0000258

35. Yu, S. Y., \& Blader, S. L. (2020). Why does social class affect subjective well-being? The role of status and power. Personality and Social Psychology Bulletin, 46, 331-348. https://doi. org/10.1177/0146167219853841

36. Wanberg, C. R., Csillag, B., Douglass, R. P., Zhou, L., \& Pollard, M. S. (2020). Socioeconomic status and well-being during COVID-19: A resource-based examination. Journal of Applied Psychology, 105, 1382-1396. https://doi.org/10.1037/apl0000831

37. Zell, E., Strickhouser, J. E., \& Krizan, Z. (2018). Subjective social status and health: A meta-analysis of community and society ladders. Health Psychology, 37, 979-987. https://doi.org/10.1037/ $\underline{\text { hea0000667 }}$

RECIBIDO: 30 de septiembre de 2021 MODIFICADO: 22 de noviembre de 2021 ACEPTADPO: 23 de noviembre de 2021 